\title{
Changes in Activities of Key Enzymes in Sugarcane Stem at Different Growing Stages
}

\author{
Rongfa Chen $^{1}$, Xing Huang ${ }^{1}$, Lihang Qiu ${ }^{1}$, Yegeng Fan ${ }^{1}$, Ronghua Zhang ${ }^{1}$, Jinlan Xie ${ }^{1}$, \\ Jianming $\mathrm{Wu}^{1,}$, Yangrui $\mathrm{Li}^{1,2}$, * \\ ${ }^{1}$ Sugarcane Research Institute of Guangxi Academy of Agricultural Sciences, Sugarcane Research Center of Chinese Academy of \\ Agricultural Sciences, Nanning, China \\ ${ }^{2}$ Guangxi Key Laboratory of Sugarcane Genetic Improvement, Key Laboratory of Sugarcane Biotechnology and Genetic Improvement \\ (Guangxi), Ministry of Agriculture, Nanning, China
}

\author{
Email address: \\ mingniansanyue@163.com (Rongfa Chen),shmilyx023@163.com (Xing Huang), liukaiqiang@gxaas.net (Lihang Qiu), \\ fanyegeng@163.com (Yegeng Fan),215100974@qq.com (Ronghua Zhang),631032669@qq.com (Jinlan Xie), \\ wujianming2004@126.com (Jianming Wu), liyr@gxaas.net (Yangrui Li) \\ ${ }^{*}$ Corresponding author
}

\section{To cite this article:}

Rongfa Chen, Xing Huang, Lihang Qiu, Yegeng Fan, Ronghua Zhang, Jinlan Xie, Jianming Wu, Yangrui Li. Changes in Activities of Key Enzymes in Sugarcane Stem at Different Growing Stages. American Journal of Plant Biology. Vol. 3, No. 2, 2018, pp. 21-28.

doi: $10.11648 /$ j.ajpb.20180302.12

Received: August 22, 2018; Accepted: September 19, 2018; Published: October 10, 2018

\begin{abstract}
Sugarcane is the most important sugar crop in China, which mainly focuses on the upper stem of the harvested land. The proper regulation of the proportion of internode elongation is the key to determine the yield and sugar. Therefore, it is of great significance to study the mechanism of the dynamic change of elongation between cane joints in order to improve the yield of sugar cane and sucrose. To investigate the biochemical mechanism of stem elongation in sugarcane, stem samples were collected at the pre-elongation stage (9-10 leaves) (Ls1), early elongation stage (12-13 leaves) (Ls2) and rapid elongation stage (15-16 leaves) (Ls3). The change trends in the activities of NAD kinase (NADK), calcium-dependent protein kinase (CDPKs), $\alpha$-mannosidase, $\alpha$-galactosidase, $\beta$-glucosidase, cellulase, xyloglucan endo-transglycosylase/hydrolase (XTH) and catalase (CAT) were completely consistent, showing rapid elongation stage $>$ early elongation stage $>$ pre-elongation stage, while the activities of $\beta$-glucosidase, peroxidase (POD) and $\alpha$-glucosidase were in opposite, and the activities of calmodulin and $\beta$-mannosidase showed the same single-peak trend, and the peak was at early elongation stage. The enzyme activities of $\beta$-galactosidase and pectinase did not show significant difference at different stages. The results indicate that the elongation of internodes was closely related to the complex physiological metabolism of sugarcane, and the key enzymes play roles at different time but $\beta$-galactosidase and pectinase have little effect on internodes elongation in sugarcane.
\end{abstract}

Keywords: Sugarcane, Stem Elongation, Enzyme Activity, Metabolism

\section{Introduction}

Sugarcane is a sugar crop whose stem is harvested, and it is the most important sugar crop in China. Sugarcane contributes to over $90 \%$ of China's total sugar production. Thus proper regulation of internode elongation is the key for improving cane yield and sugar content. The mechanisms of the dynamic change in internode elongation have been systemically studied in some plants such as bamboo and corn. The internode elongation curve and the morphological and anatomical information of internode tissue development of the bamboo and hemp bamboo have been reported [1]. It has been found that the changes of endogenous hormones, auxin and the related genes, endo- 1 , 4- $\beta$-glucanase are closely associated with internode development of bamboo shoots [2-7]. The expression patterns of time-specific and site-specific proteins were summarized and the major metabolic pathways of the differential expression have been identified [8]. Studies have 
showed that the cell length in maize internode tissues is significantly different between different stages. Differentially methylation genes are mainly involved in metabolic pathways related to plant hormone and signal transduction $[9,10]$. Other than bamboo and corn, there are few studies on the mechanisms of dynamic change in internode elongation in other plants especially there is no report on the relationship between dynamics of internode elongation and relevant enzymes in sugarcane so far. Thus, we conducted the present study to examine the difference of relevant enzymes at non-elongation, early elongation, and rapid elongation stages to provide references to further reveal the physiological mechanisms of internode elongation in sugarcane.

\section{Materials and Methods}

\subsection{Test Materials}

The experiment was conducted in the sugarcane cultivar GT42 from February to September 2016 in the intelligent greenhouse of Sugarcane Research Institute, Guangxi Academy of Agricultural Sciences.

\subsection{Sugarcane Planting and Experimental Design}

Single bud sugarcane setts were cultured, and seedlings with the same height and thickness were transplanted into the greenhouse when they grew to $8-10 \mathrm{~cm}$ in height. Three replicates were used, and each plant was used as a replicate. The samples were collected at pre-elongation (9-10 leaves) (Ls1), early elongation (12-13 leaves) (Ls2) and rapid elongation (15-16 leaves) (Ls3) stage, respectively. The internodes corresponding to leaf +2 were cut and put into liquid nitrogen, and then stored at $-80^{\circ} \mathrm{C}$ before use.

\subsection{Enzyme Assay}

\subsubsection{Signaling Factors}

Calmodulin (CAM) content, and calcium dependent protein kinase (CDPKs) and NAD kinase (NADK) activities were determined by ELISA kits

\subsubsection{Glycosidases}

$\alpha$-glycosidase $\quad(\alpha-\mathrm{GC}), \quad \beta$-glycosidase $\quad(\beta-\mathrm{GC})$, $\alpha$-mannosidase $(\alpha$-manase), $\beta$-mannosidase $(\beta$-manase), $\alpha$-galactose glucosidase ( $\alpha$-GAL), $\beta$-galactose glucosidase $(\beta-\mathrm{GAL})$ activities were assayed using spectrophotometry by the kits bought from Beijing Winter Song Boye Biotechnology Co. Ltd., following the instructions.

\subsubsection{Cell Walls Loosening Factors}

Catalase (CAT), peroxidase (POD) and wooden glucan in the glycosyl transferase/hydrolytic enzyme activities were determined by ELISA with the kits bought from Beijing Winter Song Boye Biotechnology Co. Ltd., following the instructions. Cellulase (CL) and pectinase activities were determined using spectrophotometry by the kit bought from Beijing Winter Song Boye Biotechnology Co. Ltd., following the instructions.

\subsection{Statistical Analysis}

The data mapping was completed by Graph Pad Prism 6 software, and significant difference analysis and statistical analysis were done by SPSS 20.0 software.

\section{Results}

\subsection{Dynamic Changes of Signal Transduction Factors}

Figure 1a, b showed that the change trends of NADK and CDPKs activities were consistent with, i.e., rapid elongation stage $>$ early elongation stage $>$ pre-elongation stage. The calmodulin activity showed a trend of increase at first and then decrease, but that at the early elongation and rapid elongation stages were increased by $14.69 \%$ and $7.56 \%$ respectively compared with that at non-elongation stage, and the differences were significant (Figure 1c), indicating that NADK and CDPKs may regulate internode elongation by influencing the $\mathrm{Ca}^{2}+$ signal transduction pathway, and the early elongation stage is the transition stage of CaM content which peaks at the early elongation stage and then gradually reduces to a certain level.

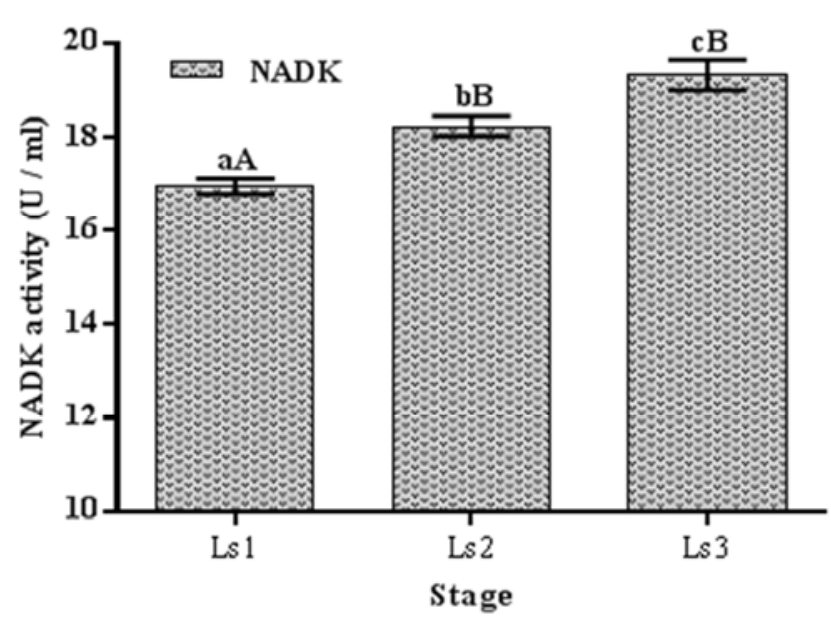

(a)

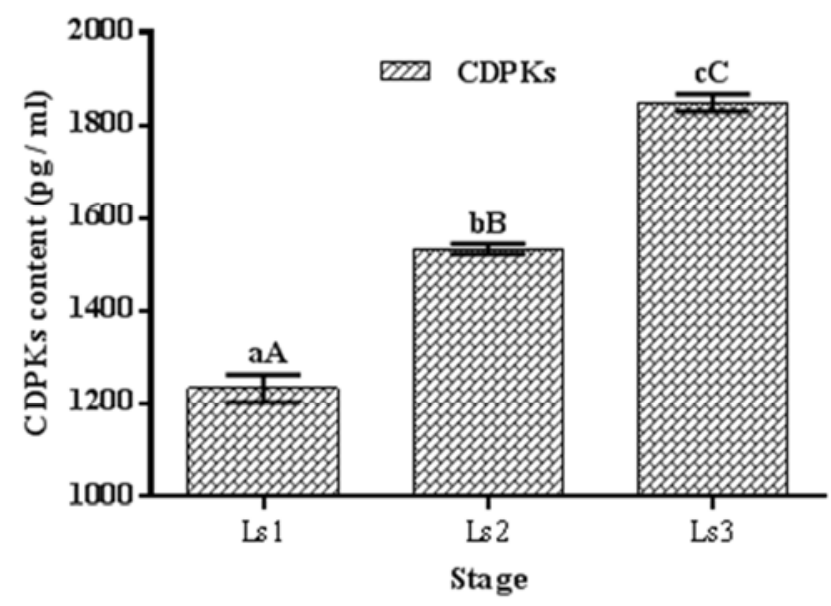

(b) 


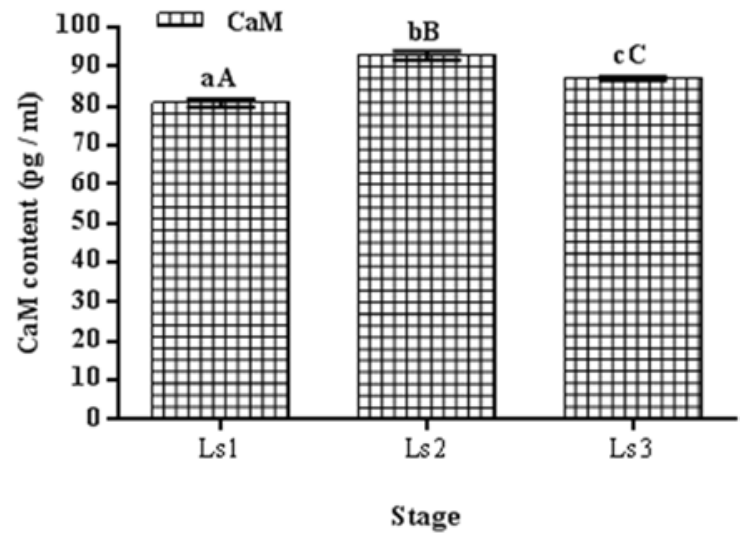

(c)

Note: Ls1: no elongation; Ls2: early elongation; Ls3: elongation stage

Figure 1. Changes of signal transduction factors NADK (a). CDPKs (b) activity and CaM content (c) in s sugarcane stem at different growing stages.

\subsection{Dynamic Change of Glycosidases}

The dynamic changes of $\alpha$-mannosidase (Figure 2a), $\beta$-glucosidase (Figure 2d) and $\alpha$-galactosidase (Figure 2e) activity showed rapid elongation stage $>$ early elongation stage $>$ pre-elongation stage, The differences of $\alpha$-mannosidase and $\alpha$-galactosidase activities between stages reached an extremely significant level (Figure $2 \mathrm{~d}$ and $2 \mathrm{e}$ ), and the $\beta$-glucosidase activities between the pre-elongation stage and early elongation stage showed no significant difference while at rapid elongation stage increased $12.60 \%$ and $14.43 \%$ compared with those at early elongation stage and pre-elongation stage respectively (Figure 2e). However, the changes in the activity of $\alpha$-glucosidase showed an opposite trend, which decreased by $15.20 \%$ at early elongation stage compared with that at pre-elongation stage, and then became stabile afterward (Figure 2c). The $\beta$-galactosidase activity showed a single peak in its dynamic change, the peaked appeared at early elongation stage and then decreased afterward, but increased by 32.42 and $11.90 \%$ respectively at the early and rapid elongation stages compared with that at non-elongation stage (Figure 2e). The differences of $\beta$-galactosidase between different stages were not significant (Figure 2f).

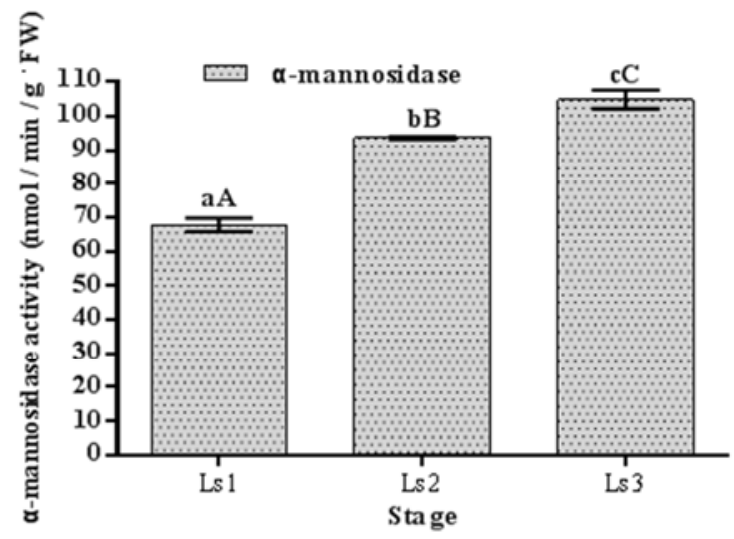

(a)

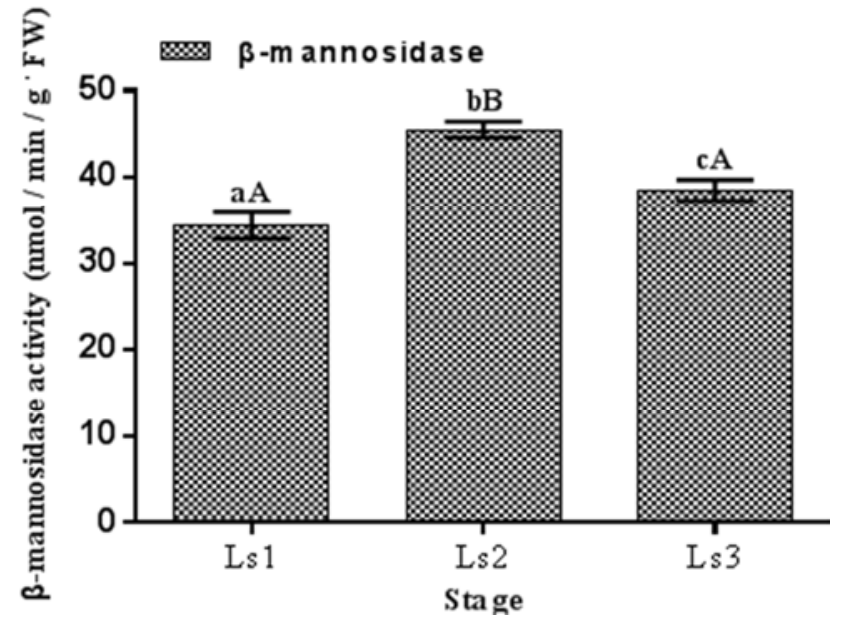

(b)

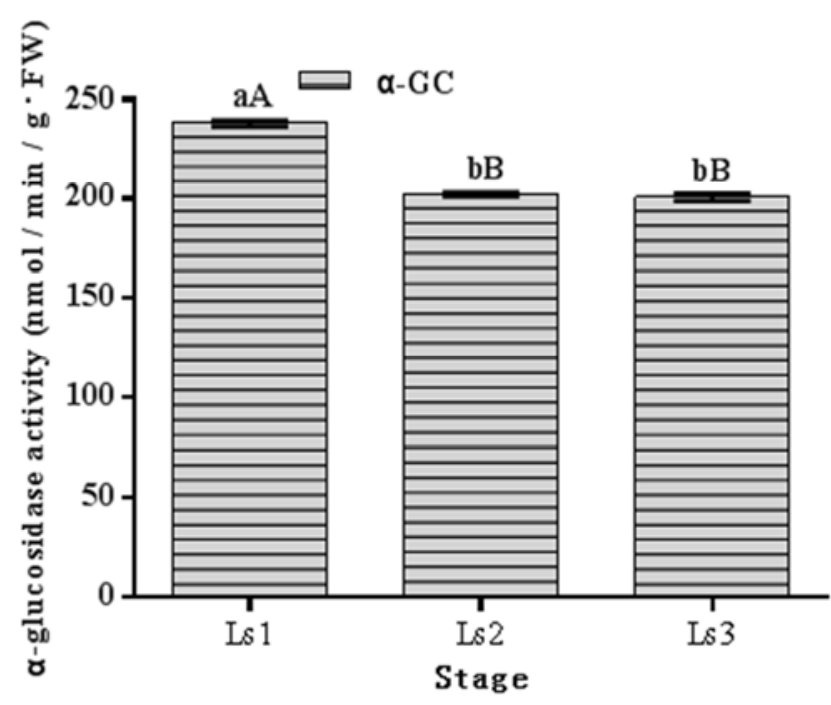

(c)

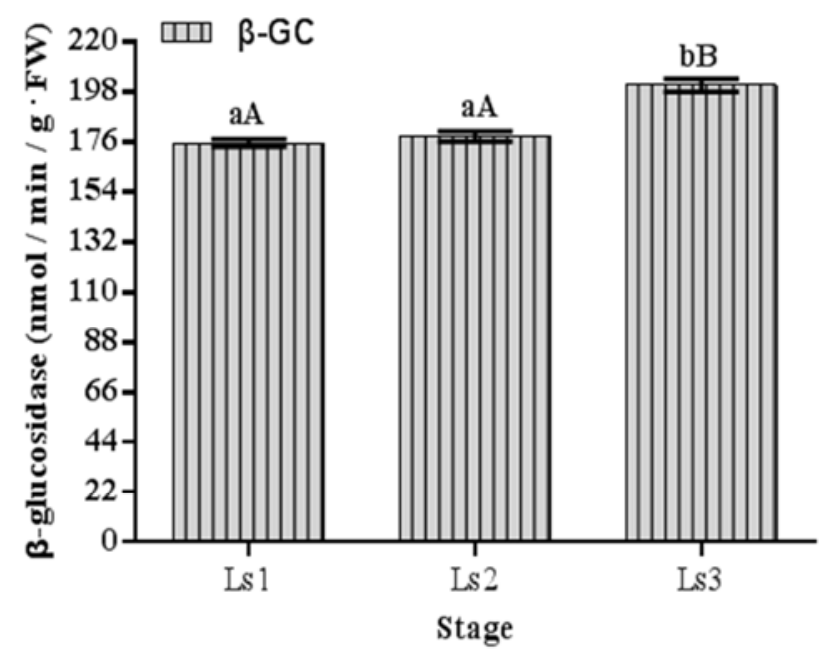

(d) 


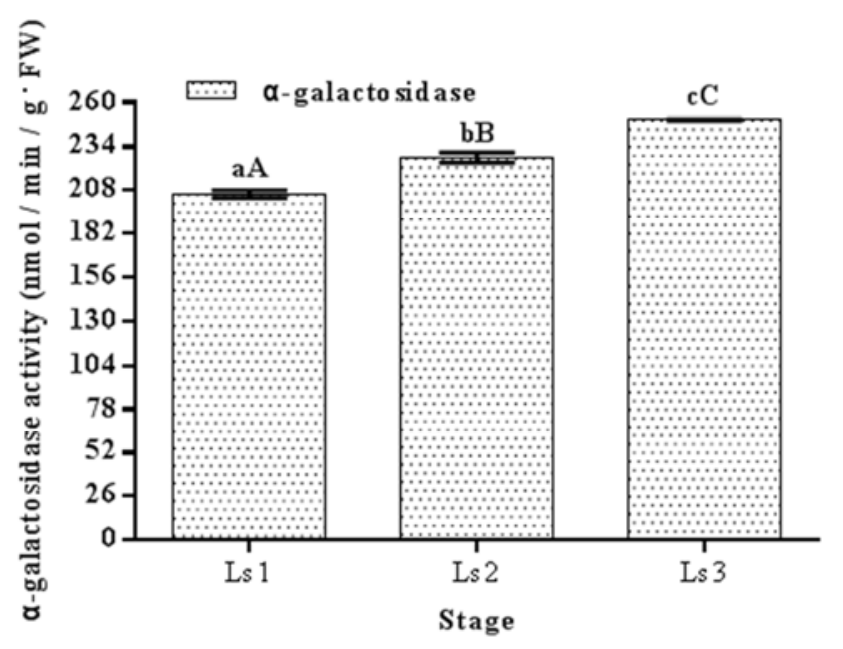

(e)

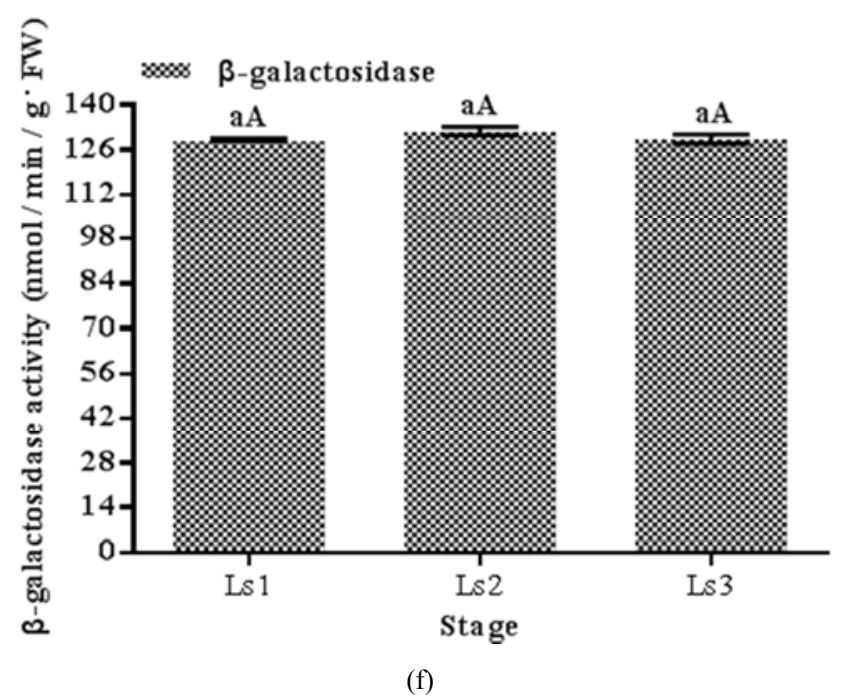

Note: Ls1: no elongation; Ls2: early elongation; Ls3: elongation stage

Figure 2. Changes of $\alpha$-mannosidase (a), $\beta$-mannosidase (b), $\alpha$-glucosidase $(\alpha-G C)(c), \beta$-glucosidase $(\beta-G C)(d)$, - $\alpha$ galactosidase $(\alpha-G A L)(e)$ and $\beta$-galactosidase $(\beta-G A L)(f)$ activities in stem of sugarcane at different growing stages

\subsection{Dynamic Change of Cell Wall Loosening Factors}

The cellulase and XTH activities were completely consistent, which increased with the elongation of sugarcane internodes, and all the differences were extremely significant (Figure $3 \mathrm{a}$ and e). Figure $3 \mathrm{~b}$ showed that the pectinase activity and it was not significantly different at different stages, indicating that it did not play a role in the elongation of internodes. However, the POD activity exhibited an opposite trend, i.e., pre-elongation stage $>$ early elongation $>$ rapid elongation stage and the differences were extremely significant (Figure 3c). The CAT activity showed the increasing trends i.e., rapid elongation stage $>$ early elongation stage, and the differences were extremely significant (Figure 3d).

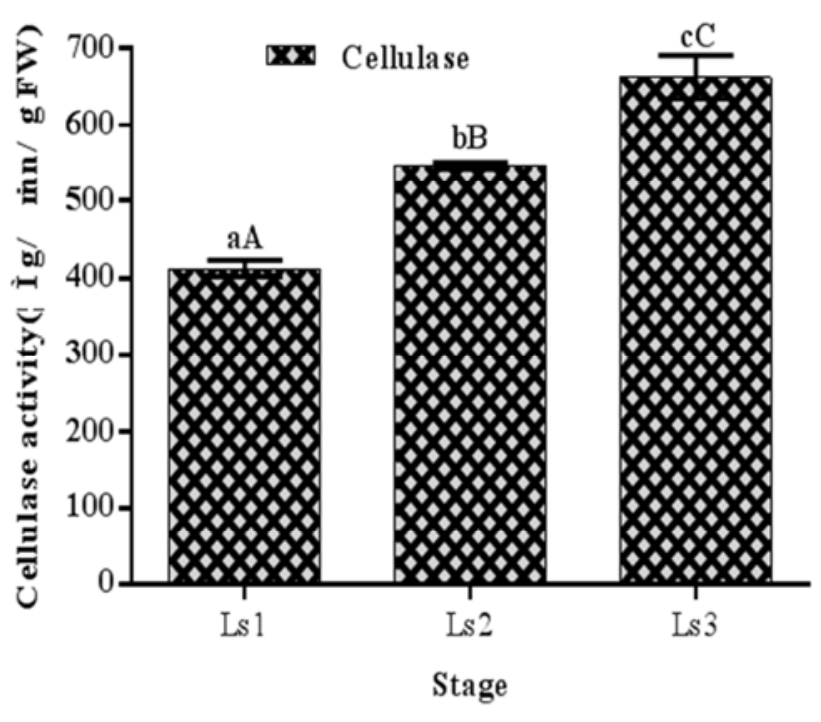

(a)

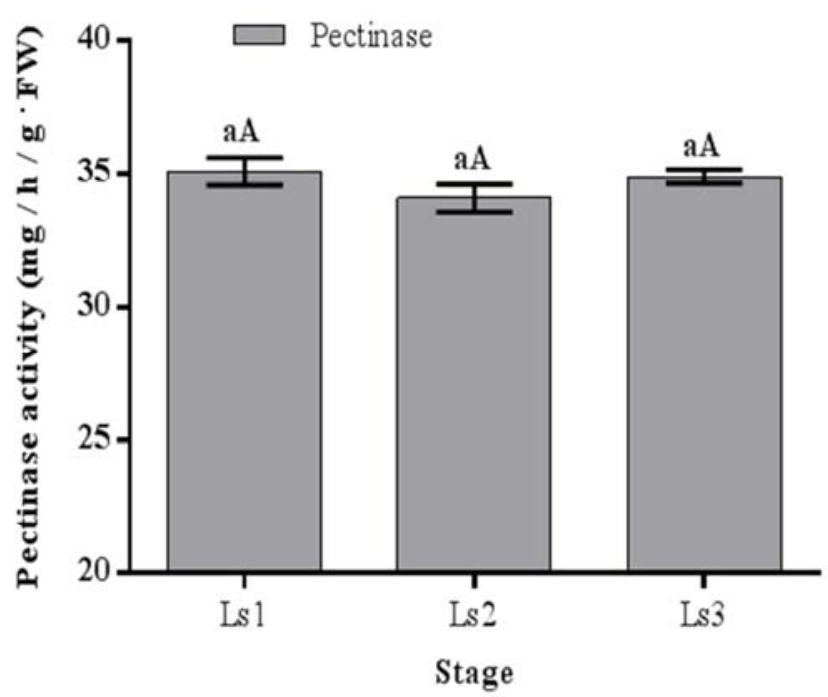

(b)

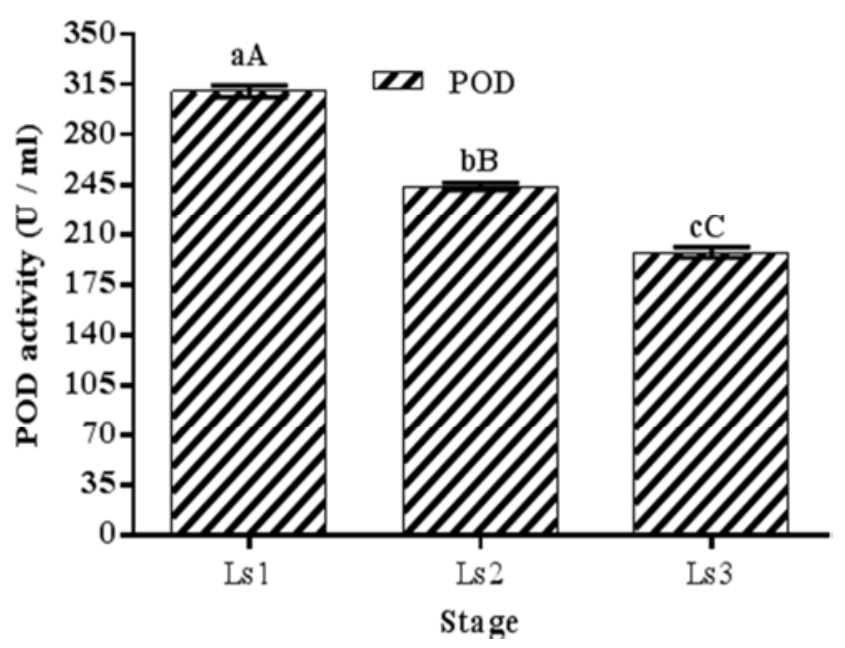

(c) 


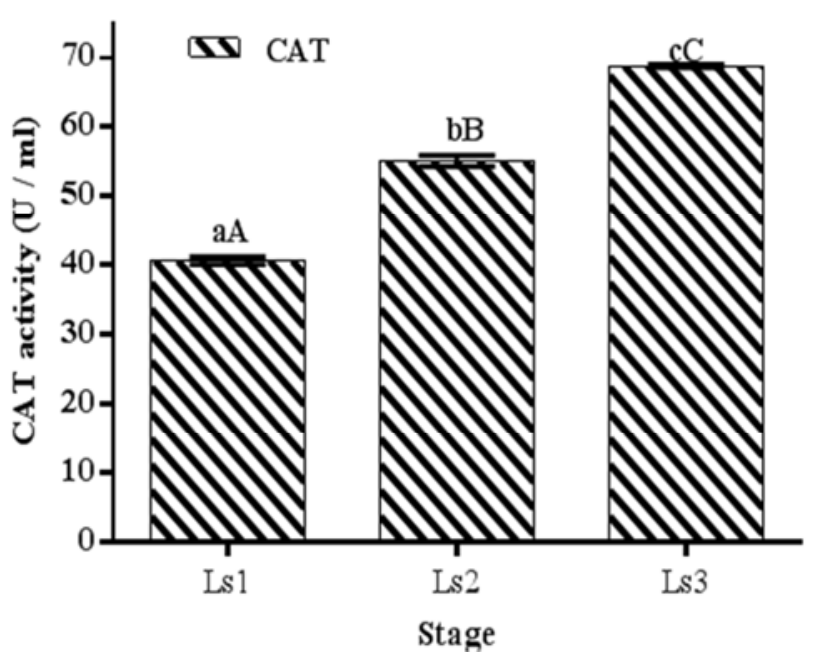

(d)

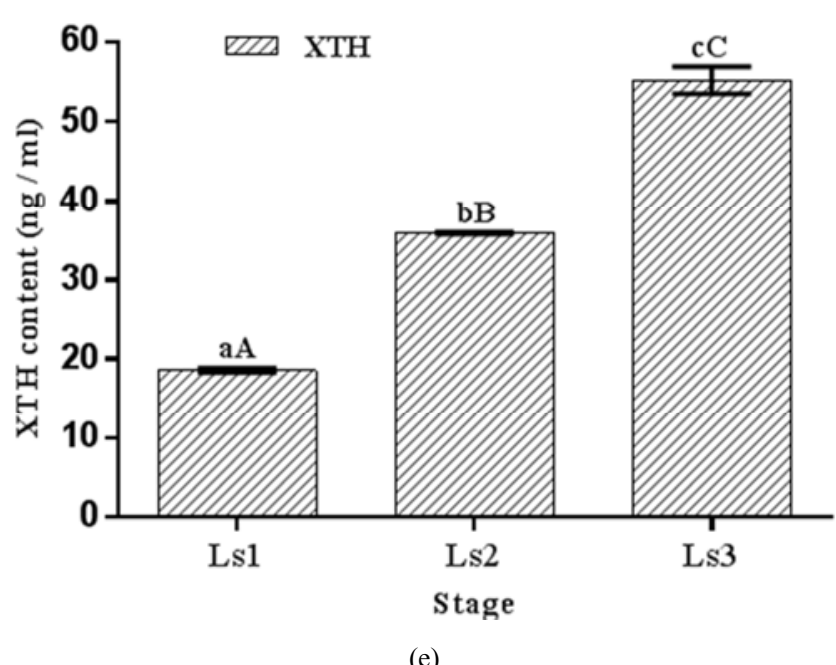

Note: Ls1: no elongation; Ls2: early elongation; Ls3: elongation stage

Figure 3. Changes of XTH content of different sugarcane growing stage.

\section{Discussion}

\subsection{Relationship Between Signal Transduction Factors and Elongation of Sugarcane Internode}

CaM has a wide range of physiological functions, and it involves in regulation of many physiological functions. Previous studies have indicated that exogenous CaM can promote pollen germination and pollen tubes elongation, and can induce physiological functions of specific genes [11-13]. In this study, CaM activity showed an increased trend with the elongation of sugarcane internodes, and reached a peak at the early elongation stage but was stabilized afterward, indicating that $\mathrm{CaM}$ can promote the elongation and the early elongation stage was the key stage for its function. It is possible that CaM dynamically influences the $\mathrm{Ca}^{2+}$ concentration in cytoplasm, ultimately leading to the stimuli-specific physiological response to promote the elongation. This is similar to the results of previous studies on the promotion of pollen tube elongation by CaM.
NADK is one of the important enzymes of CaM activated by $\mathrm{Ca}^{2+}$ in plant cells. Studies have shown that in the root elongation process of pea seedling, NADK and CaM activity change over the elongation process [14]. Wang and Pan previously reported that when red light treatment induces an increase of mitochondrial NADK in mung bean hypocotyl cuttings, the content of activated $\mathrm{CaM}$ in the cytoplasm also increases correspondingly. Up to now, there is no direct study to prove the relationship between NADK and internode elongation. The results of this study showed that, the activity of NADK in young stems of sugarcane increased significantly with the elongation of sugarcane internodes, indicating that NADK may regulate the elongation of sugarcane internodes by affecting CaM content or active oxygen species in sugarcane.

Plant CDPKs participate in the regulation of plant cell actin tension, stomatal openness and regulation of plant growth and development [16-17]. CDPKs genes belong to a gene family, in which the expression patterns of different genes are different, some genes encoded by CDPKs are negatively correlated with elongation [18]. The results of this study indicated that the activities of CDPKs in the young stems of sugarcane increased with the elongation of internodes, indicating that CDPKs positively regulate the elongation of internodes in sugarcane, which may reflet different functions of different CDPKs, and the temporal and special differences between functions of homologous factors in the same signaling pathway.

\subsection{Relationship Between Glycosidases and Elongation of Sugarcane Internode}

Loosening of the cell wall is necessary for cell elongation, and glycosidases are associated with loosening and elongation of cell walls [19]. Previous studies have indicated that $\alpha$-galactosidase may be related to cell growth process, and $\beta$-galactosidase may be related to cell wall loosening [20]. The results of this study also indicated that the activity of $\alpha$-galactosidase, $\alpha$-mannosidase, $\quad \beta$-glucosidase and $\beta$-mannosidase increased with the elongation of sugarcane internodes, but the degrees and trends of the increase were different, and became stabile after a significant decrease from the pre-elongation stage to the early elongation stage; while $\beta$-galactosidase was not associated with elongation growth, indicating that $\alpha$-galactosidase, $\alpha$-mannosidase, $\beta$-glucosidase and $\beta$-mannosidase may positively regulate the elongation of sugarcane internodes through affecting the loosening of cell walls, but the duration of their function and their effects are different. However, the $\alpha$-glucosidase seemed to negatively regulate the elongation of sugarcane internodes, and the effects are mainly exerted from the pre-elongation stage to the early elongation stage, while $\beta$-galactosidase was not associated with the elongation growth.

\subsection{Relationship Between Cell Wall Loosening Factors and Elongation of Sugarcane Internode}

Cellulase and pectinase are important hydrolases in cell wall. Studies have indicated that the improvement of cell 
elongation is due to that IAA promotes the secretion of $\mathrm{H}^{+}$in the cell wall and activates cellulase which in turn induces degradation, causing cell wall loosening [21]. The results of this study showed that the activity of cellulase significantly increased, and pectinase showed no significant difference in different stages of internode elongation, indicating that cellulase leads to cell wall loosening possibly through affecting XG degradation and further affects the elongation of sugarcane internodes, while pectinase has little effect of the enzyme on internode elongation.

XTH is widely present in various tissues and cells in plants, and is one of the key enzymes in plant cell wall remodeling [22]. Studies have indicated that xyloglucan endotransglucosylase (XET) is important for loosening cell walls and plays a major role in cell elongation or internode elongation [23-34]. The results in this study also showed that XTH activities increased significantly with the elongation of sugarcane internodes, indicating that the XTH plays an important role in regulating the elongation of sugarcane internode.

Reactive oxygen species (ROS) are important regulators of plant growth and development, and are directly involved in plastic change of cell wall loosening and hardening, affecting characteristics of cell walls, and further regulating cell growth [35-37]. Both catalase and peroxidase are closely related to ROS [38-39]. Hydrogen peroxide promotes cell or tissue elongation [39-40]. These results are indicating that hydrogen peroxide promotes cell or tissue elongation. The results in this study also confirmed this view, during the elongation stage, higher CAT activity was induced in sugarcane to eliminate the large amount of free radicals and improve internode elongation. Peroxidase has the function of oxidative degradation of indoleacetic acid [41-42], so the ratio of peroxidase vs. indoleacetic acid is critical to the physiology of plants [43]. Relevant studies also indicated that the peroxidase activities in elongating internodes of sorghum, wheat, maize and sugarcane are negatively correlated with plant heights [43-45]. The results of this study are consistent with the previous studies.

\section{Conclusion}

In the present study, we found that the activities of NADK, calcium-dependent protein kinase (CDPKs), $\alpha$-mannosidase, $\alpha$-galactosidase, $\beta$-glucosidase, cellulase, XTH, and catalase have significant positive correlations with the elongation of sugarcane internode, which indicate that they play an important positive regulatory role in the elongation of sugarcane internodes. The dynamic changes of the activities of POD and $\alpha$-glucosidase were in opposite, both of them showed significant negative correlation with the elongation of sugarcane internode, indicating that they play a negative regulatory role, but the acting time and the effects are significantly different. POD affects the whole process, while $\alpha$-glucosidase has greater effect at the early elongation stage. The dynamic change of the activities of calmodulin and $\beta$-mannosidase were completely consistent, showing a single peak at the early stage of elongation, indicating that, they play a key role in the start of the elongation of sugarcane internode. However, the regulatory modes of these metabolic enzymes on the elongation of sugarcane internode need to be further studied.

\section{Acknowledgements}

RF-C has participated in the design of the experiments, the analytical determinations and the writing and discussion of the manuscript. $\mathrm{XH}$ has participated in the design of the experiments and the analytical determinations. LH-Q has participated in the design of the experiments, the analytical determinations and the writing and discussion of the manuscript. YG-F, R H-Z, J L-X has participated in the design of the experiments. the analytical determinations and the writing and discussion of the manuscript. JM-W has participated in the design of the experiments and the analytical determinations. YR-L has participated in the design of the experiments and the analytical determinations. All authors revised the manuscript.

This work was supported in part by National Natural Foundation of China (31360312), Guangxi science and technology development program projects (Gui Ke Gong 1598006-1-2E), Guangxi natural science foundation project (2015GXNSFDA139011, 2017GXNSFBA198050), Guangxi Special Funds for Bagui Scholars (2013-03), Guangxi special fund for scientific base, talent (GKAD17195100), Project of Guangxi Sugarcane Innovation team of National Modern Agriculture Industry Technology System (gjnytxgxcxtd-03) and Guangxi Academy of Agricultural Sciences Basic Research Business Special (Gui nong ke 2017YM03).

\section{References}

[1] Li, X., Z. H. Guo (2014). A Pilot Study on Internode Elongation in a Paleotropical Bamboo, Dendrocalamus latiflorus (Poaceae: Bambusoideae). Plant Diversity and Resources 36(1):22-28.

[2] Wang, H. Y., K. Cui , C. Y. He, Y. F. Zeng, S. X. Liao, and J. G. Zhang (2015). Endogenous hormonal equilibrium linked to bamboo culm development. Genetics Molecular Research 14(3):11312-11323.

[3] He, C. Y., K. Cui, J. G. Zhang, A. G. Duan, and Y. F. Zeng (2013). Next-generation sequencing-based mRNA and microRNA expression profiling analysis revealed pathways involved in the rapid growth of developing culms in Moso bamboo. BMC Plant Biology 13(1):1-14.

[4] Peng, Z. H., CH. L. Zhang, Y. Zhang, T. Hu, S. H. Mu, X. P. Li, and J. Gao (2013). Transcriptome sequencing and analysis of the fast growing shoots of Moso bamboo(Phyllostachys edulis) PLOS One 8(11):e78944.

[5] Gao, J., Y. Zhang, C. L. Zhang, F. Y. Qi, X. P. Li, SH. H. Mu, and ZH. H. Peng (2014). Characterization of the floral transcriptome of Moso bamboo (Phyllostachys edulis) at different flowering developmental stage by transcriptome sequencing and RNA-seq analysis. PLOS One 9(6): e98910. 
[6] Zhang, Y., G. Tian, H. P. Lu, R. Hong, T. Hu, and Q. R. Guo.2015. Transcriptome characterization of phyllostachys edulis'Pachyloen'shoots in different solar terms. Acta Agriculturae Universitatis Jiangxiensis 37(3):466-474.

[7] Zhou, M. B., Y. Zheng, L. Z. Liu, X. W. Xia, D. Q. Tang, Y. Fu, and $M$. Chen (2016). Endo-1,4- $\beta$-glucanase gene involved into the rapid elongation of Phyllostachys heterocycla var. pubescens. Trees 30(4):1259-1274.

[8] Cui, K., C. Y. He, J. G. Zhang, A. G. Duan, and Y. F. Zeng (2012). Temporal and spatial profiling of Internode elongation associated protein expression in rapidly growing culms of bamboo. Journal of Proteome Research 11: 2492-2507.

[9] Li, P (2012). DNA methylation analysis and QTL mapping for internode elongation of Maize (Zea mays L.). Shijiazhuang, Hebei: Agricultural University of Hebei.

[10] Zhang, K (2014). Methylation and expression analysis of genes involved in the Internode elongation in Maize. Wuhan, Hubei: Huazhong Agricultural University.

[11] Ma, L. G., and D. Y. Sun (1996). The extracellulr reaction sites of calmodulin on pollen and pollen tubes of Hippeastrumrurilum. Progress in Natural Science (6):505-509.

[12] Cui, S. J., H. H. Wang, L. G. Ma, and D. Y. Sun (1998). The effects of extracellular calmodulin of style and pollen on pollen germination and pollen tube growth. Plant Physiology Journal 24(4):320-326.

[13] Yang, H. Y (1999). The role of calcium in the fertilization process in flowering plants. Chinese Bulletin of Botany 41(10):1027-1035.

[14] Allan, E., and A. Trewavas (1985). Quantitative changes in calmodulin and NAD kinase during early cell development in the root apex of Pisum sativum L. Planta 165:493-501.

[15] Wang, X. J., and R. Z. Pan (1993). Effects of red light on calcium accumulation, activities of ATPase and NAD kinase in mitochondria isolated from hypocotyls segments of Mung Bean. Plant Physiology Journal 19(1):71-76.

[16] Liu, G. S., and J. Chen (2003). Roles of calcium dependent protein kinases (CDPKs) in plant calcium signal transduction. Chinese Bulletin of Botany 20:160-197.

[17] Huang, J. Z., S. C. Hardin, and S. C. Huber (2001). Identification of a novel phosphorylation motif for CDPKs: Phosphorylation of synthetic peptides lacking basic residues at P-3/P-4. Archives of Biochemistry Biophysics 393:61-66.

[18] Breviario, D., L. Morello, and S. Giani (1995). Molecular cloning of two novel rice cDNA sequences encoding putative calcium- dependent protein kinase. Plant Molecular Biology 27(5):953- 967.

[19] Li, L. C., X. C. Wang (1998). The relationship between plant cell elongation and wall properties under water deficits. Plant Physiology Communications 34(3):161- 167(in Chinese).

[20] Li, L. C., X. C. Wang. 1996. Effects of water deficit on plant cell wall and its relation to cell elongation. Plant Physiology Communications 32(5):321-327.

[21] Hayashi, T., Y. S. Wong, and G. Maclachlan (1984). Pea xyloglucan and cellulose: II . Hydrolysis by pea endo-1,4-glucanases. Plant Physiology 75:605-610.
[22] Xuan, Y., H. F. Zhao, X. Y. Guo, J. Ren, Y. Wang, and B. Y. $\mathrm{Lu}$ (2016). Plant cell wall remodeling enzyme xyloglucan endotransglucosylase/hydrolase (XTH). Chinese Agricultural Science Bulletin 32(8):83-88.

[23] Fry, S. C. (2000). The Growing Plant Cell Wall: Chemical and Metabolic Analysis. New Jersey: The Blackburn Press, Caldwell.

[24] Cosgrove, D. J (2005). Growth of the plant cell wall. Nature Reviews Molecular Cell Biology 6:850-861.

[25] Potter, I., and S. C. Fry (1993). Xyloglucan endotransglycosylase activity in pea internodes. Plant Physiology 103(1):235-241.

[26] Potter, I., and S. C. Fry (1994). Changes in xyloglucan endotransglycosylase (XET) activity during hormone- induced growth in lettuce and cucumber hypocotyls and spinach cell suspension cultures. Journal of Experimental Botany 45:1703-1710.

[27] Smith, R. C., and P. R. Matthews (1996). The regulation of leaf elongation and xyloglucan endotransglycosylase by gibberellin in 'Himalaya' barley (Hordeum vulgare L). Journal of Experimental Botany 47(302):1395-1404.

[28] Michailidis, G, A. Argiriou, N. Darzentas, and A. Tsaftaris (2009). Analysis of xyloglucan endotransglycosylase/hydrolase (XTH) genes from allotetraploid (Gossypium hirsutum) cotton and its diploid progenitors expressed during fiber elongation. Journal of Plant Physiology 166(4): 403-416.

[29] Jimenez, T., I. Martin, E. Labrador, and B. Dopico (2006). The immunolocation of a xyloglucan endotransglucosylase/hydrolase specific to elongating tissues in Cicer arietinum suggests a role in the elongation of vascular cells. Journal of Experimental Botany 57(15): 3979-3988.

[30] Hernandez, N. J., I. Martin, E. Labrador, and B. Dopico (2010). The immunolocation of XTH1 in embryonic axes during chickpea germination and seedling growth confirms its function in cell elongation and vascular differentiation. Journal of Experimental Botany 61(15): 4231-4238.

[31] Hyodo, H., S. Yamakawa, Y. Takeda, M. Tsuduki, A. Yokota, K. Nishitani, and T. Kohchi (2003). Active gene expression of a xyloglucan endotransglucosylase/hydrolase gene, XTH9, in infolorescence apices is related to cell elongation in Arabidopsis thaliana. Plant Molecular Biology 52(2):473-482.

[32] Shin, Y. K., H. K. Yum, E. S. Kim, H. Cho, K. M. Gothandam, J. Hyun, and Y. Y. Chung (2006). BcXTH1, a Brassica campestris homologue of arabidopsis XTH9, is associated with cell expansion. Planta 224(1):32-41.

[33] Yokoyama, R., J. K. C. Rose, and K. Nishitani (2004). A surprising diversity and abundance of xyloglucan endotransglucosylase/hydrolases in rice. Classification and expression analysis. Plant Physiology 134(3): 1088-1099.

[34] He, H., R. Serraj, and Q. Yang (2009). Changes in Os XTH gene expression, $\mathrm{ABA}$ content, and peduncle elongation in rice subjected to drought at the reproductive stage. Acta Physiologiae Plantarum 31(4): 749-756.

[35] Schopfer, P (1996). Hydrogen peroxide-mediated cell-wall stiffening in vitro in maize coleoptiles. Planta 199:43-49. 
[36] Fry, S. C (1998). Oxidation scission of plant cell wall polysaccharides by ascorbate induced hydroxyl radicals. Biochemical Journal 332:507-515.

[37] Neill, S. J., R. Desikan, A. Clarke, R. D. Hurst, and J. T. Hancock (2002). Hydrogen peroxide and nitric oxide as signaling molecules in plants. Journal of Experimental Botany 53(372):1237-1247.

[38] Drążkiewicz, M., E. Skórzyńska-Polit, and Z. Krupa (2004). Copper-induced oxidative stress and antioxidant defence in Arabidopsis thaliana. Bio Metals 17(4):379-387.

[39] Wu, J. M., Y. R. Li, A. Q. Wang, Y. Yang, and L. T. Yang (2010). Relationship between gibberellin-induced internode elongation and related enzyme activities in sugarcane. Acta Botanica Boreall-Occidentalla Sinica 30(5):0962-0967.

[40] Bailly, C. 2004. Active oxygen species and antioxidants in seed biology. Seed Science Research 14(2):93-107.

[41] Intapruk, C., K. Yamamoto, M. Sekine, M. Takano, and A. Shinmyo (1994). Regulatory sequences involved in the peroxidase gene expression in Arabidopsis Thaliana. Plant Cell Reports 13(3):123-129.
[42] Liu, Z. H., and M. J. Ger (1997). Changes of enzyme activity during pollen germination in maize, and possible evidence of lignin synthesis. Functional Plant physiology 24(3):329-335.

[43] Liang, Y. R., X. H. H, Y. L. Zhang, and X. P. Liu (2003). Prdgress on physiological function research of plant preoxidase. Journal of Inner Mongolia Agricultural University 24(2):110-113.

[44] Zhou, R. G, Y. S. Han, L. F. Yan (1983). The relationship between plant height and peroxidase. Acta Agronomica Sninica 9(4):267-273.

[45] Liang, G. H., K. C. Lee, K. Chung, Y. T. Liang, and B. A. Cunningham (1977). Regulation of internnode length by peroxidase enzymes in grain sorghum. Theoretical and Applied Genetics 50(3):137-146. 